\title{
Management of postsurgical pain in the community
}

\section{SUMMARY}

Following surgery there is often a need for ongoing pain management after the patient is discharged from hospital. This can be made easier if the patient has an appropriate discussion before leaving hospital about what pain they can expect, and they are given a management plan.

Paracetamol and non-steroidal anti-inflammatory drugs are suitable for most patients. Drugs with a short half-life, such as ibuprofen, may need to be taken regularly.

Short-acting opioids can have a short-term role, providing guidelines are followed. There is a predictable period of time after surgery when the benefit of an opioid is expected to be maximised before harmful adverse effects will dominate.

Gabapentinoids are useful for neuropathic pain, but have a limited role in nociceptive pain. Like opioids, they have a risk of misuse.

The surgeon should be consulted if the patient develops new pain or the postoperative pain becomes more severe.

Most postsurgical pain will resolve within three months. If not, it is deemed persistent pain that may warrant specialist assessment.

\section{Introduction}

After a patient is discharged from hospital the management of postsurgical pain in the community can be challenging. The degree of difficulty this poses depends on the patient's management before they leave hospital.

On returning home, it is important the patient has realistic expectations of what to expect regarding their pain. This can be facilitated by an appropriate discussion with the surgical or pain management team before discharge. Preferably, the patient is given a written pain management plan that can be followed after leaving hospital, with realistic goals as to when they can return to physical activity or work. Communicating these plans with the patient's local doctor is critical. Patients who still have moderate pain who are discharged with long-acting opioids, without a plan, or any expectation of what to expect in terms of ongoing management, are at a significant disadvantage.

On discharge from hospital the quantity of analgesic drugs should be limited to the equivalent of 2-3 days supply. This encourages the judicious use of the drugs and ensures early contact with their GP to obtain any continuing supply.

\section{Incidence of pain}

There have been many well-conducted studies on the incidence of postsurgical pain following discharge from hospital. One major review found the incidence of persistent pain was $13.6 \%$ four months after hernia repair, $11.8 \%$ after vaginal hysterectomy and $25.1 \%$ after abdominal hysterectomy. At one year these figures had fallen to $6.2 \%, 4.1 \%$ and $9.9 \%$.

Other studies enable the likelihood of an increased incidence of persistent pain to be predicted. This is based on both surgical and patient factors (see Boxes 1 and 2). ${ }^{2,3}$

\section{'Red flags'}

While some ongoing pain is to be expected after surgery, clinicians should be alert for the appearance of new pain, or pain with increasing severity. This may herald a 'red flag' that might be associated with a complication from the procedure and necessitates referral back to the surgeon. Importantly, ask if the pain has been severe enough to prevent sleep or impede return to some degree of function.

It is important not to miss the emergence of neuropathic pain, which usually responds poorly to conventional analgesics. Pain that occurs within a

\section{Ross MacPherson}

Clinical Professor

\section{Gavin Pattullo}

Director, Acute Pain Service Department of Anaesthesia and Pain Management, Royal North Shore Hospital, Sydney

\section{Keywords}

gabapentinoids, nonsteroidal anti-inflammatory drugs, opioids,

postoperative pain

Aust Prescr 2020;43:191-4

https://doi.org/10.18773/ austprescr.2020.062 
circumscribed nerve distribution, exhibits hyperalgesia or allodynia, or is described as 'shooting' or like an 'electric shock' by the patient should arouse suspicion. This is particularly common following certain types of surgery, such as mastectomy or thoracotomy. Neuropathic pain requires a different approach to treatment, so it is crucial that it is detected at an early stage.

\section{A systematic approach}

Analgesics are a key component of postsurgical pain management. Using a multimodal approach to analgesia can both maximise the response to medicines and limit the use of opioids.

The prescriber must remember that, with the exception of paracetamol and non-steroidal antiinflammatory drugs (NSAIDs), all of the commonly used analgesics can have significant depressant effects on the central nervous system. While tolerance no doubt develops, drowsiness and effects on highercentre functions may impede the patient's capacity to drive or to return to work.

\section{Paracetamol}

Paracetamol is a useful starting point for any analgesic plan. If the pain is persistent, the patient should be encouraged to take paracetamol regularly rather than as needed.

\section{Box 1 Surgical procedures associated with a high incidence of persistent postoperative pain ${ }^{2}$}

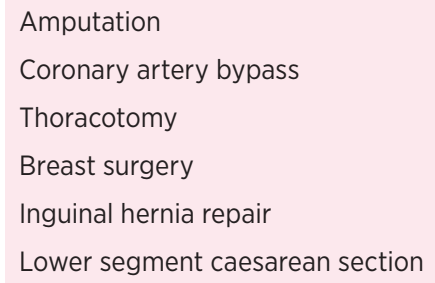

\section{Box 2 Patient factors associated with an increased incidence of postoperative pain ${ }^{3}$}

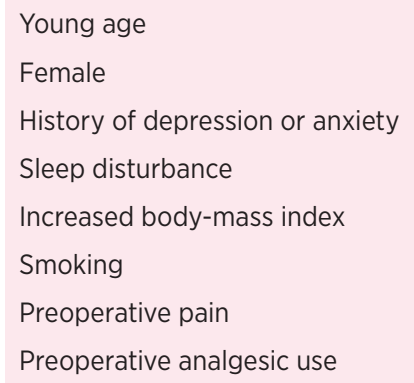

\section{Non-steroidal anti-inflammatory drugs}

For uncomplicated nociceptive pain, NSAIDs are now first line unless contraindicated. Relative contraindications include previous gastrointestinal adverse events, kidney disease, history of cardiovascular disease, older age, and an increased risk of bleeding. The risk of adverse effects needs to be carefully monitored, especially because their incidence appears to be correlated with increases in the dose and duration of treatment.

Although these drugs can be purchased over-thecounter, they are effective analgesics - a fact that often needs to be reinforced with patients. NSAIDs are particularly useful for pain precipitated by movement. ${ }^{4}$ While there may be some reticence by surgeons to prescribe these drugs in the very early postoperative period, because of concern about an increased risk of bleeding, this should not be a reason to withhold NSAIDs by the time the patient is rehabilitating.

There seems to be little to differentiate these drugs in terms of efficacy apart from their COX-2 selectivity and pharmacokinetics. Drugs with short half-lives such as ibuprofen or diclofenac need to be taken regularly throughout the day which is a possible disadvantage compared to drugs with long half-lives such as meloxicam. However, drugs with short half-lives have a more rapid onset of action and, should any adverse effects occur, can be quickly discontinued.

COX-2-specific drugs provide a similar level of analgesia, but the incidence of gastrointestinal adverse effects may be lower. While it is a long-held belief that NSAIDs need to be taken with food, recent studies have shown that this does not reduce the rate of gastrointestinal adverse effects. Indeed, taking them on an empty stomach results in higher blood concentrations and improved analgesia.

\section{Opioids}

Opioids should have a limited role once the patient is discharged from hospital. If they are used in initial pain management, only immediate-acting products should be prescribed, unless the patient was taking sustained-release drugs before admission for surgery. ${ }^{5}$

The opioid crisis has reinforced the need for more considered prescribing of pure opioid agonists with a clear understanding for both the doctor and the patient of what role the drugs play. While opioids relieve pain they should not be seen as first-line analgesics. They are best viewed as rescue medicines. There are some general considerations that should apply: 
1. Opioid use comes at a small risk of significant harm to the patient. Based on recent data, for approximately every 4000 Australians prescribed opioids, there will be one death in the population per year. ${ }^{6,7}$

2. A patient who reports feeling the expected sensations after surgery, who is already taking non-opioid analgesics (e.g. paracetamol plus an NSAID) and who is coping, is unlikely to gain much benefit from the addition of an opioid.

3. When opioids are indicated and a benefit is seen, the dose should be titrated to effect with close monitoring.

4. There is a predictable period of time after an acute injury when the benefit of an opioid is expected to be maximised before harmful adverse effects will dominate. It is helpful if the surgeon can inform the patient's GP what this duration is likely to be. For most types of surgery it is a few days.

5. Opioids are weaned at a rate which matches the resolution of the source of pain. After short-term use of a few days, opioids are commonly weaned rapidly without adverse consequences. Once their use extends into weeks, weaning is generally slower as a compromise between clinical need and patient tolerance. As a rough guide, reduce the dose by approximately $25-40 \%$ every few days. The use of drugs with a short half-life, for the shortest possible time, should be the aim.

With regard to the choice of drug, pure opioid agonists have largely replaced the use of the prodrug codeine. Codeine has an unpredictable effect owing to genetically determined variations in metabolism, and when used alone has negligible analgesic properties.

In adults, determining the opioid dose is based more on the age of the patient and presence of debilitating comorbidities, rather than body weight as is often used. Indeed, at the upper extreme of size, owing to the likelihood of obstructive sleep apnoea, weightbased dosing should not be used.

Tapentadol, which is available in immediate-release and sustained-release formulations, is appropriately classified as an opioid, but has a dual mechanism of action which also involves noradrenergic pathways. It has a relatively weak opioid effect and this is reflected in its lower incidence of morphine-like adverse effects.

Tramadol can be useful in the early management of postoperative pain. It acts via opioid receptor stimulation and augmentation of noradrenaline (norepinephrine) and serotonin pathways. Some studies have suggested tramadol may be beneficial in neuropathic pain states. However, the response to tramadol can be mixed, most likely as a result of the complex pharmacokinetic polymorphism associated with its metabolism. ${ }^{8}$ This probably explains its failure to elicit a useful response in a subgroup of patients.

\section{Gabapentinoids}

While research suggests that pregabalin and gabapentin are useful for neuropathic pain states, reports of their efficacy in postsurgical pain have been mixed. . $^{910}$ Furthermore, there are significant risks associated with long-term prescribing:

- central nervous system depressant effects will be enhanced by concomitant use of opioids

- the incidence of adverse effects, especially somnolence and dizziness, is significant

- $\quad$ there is evidence of dependence."

\section{When to refer}

Most pain should resolve within three months. The emergence of red flags after discharge requires prompt consultation with the surgeon.

The International Association for the Study of Pain has defined chronic postsurgical pain as a condition that exists when the pain:

- $\quad$ persists three months after surgery

- was not present before surgery or was of a different character

- originates from the surgical site, or a referred area

- does not have any explainable cause. ${ }^{12}$

When pain persists for more than three months after discharge, or the patient has been unable to return to an expected level of functioning, further assessment either by the treating surgeon or a specialist pain clinic is warranted.

\section{Conclusion}

Postsurgical pain is common and a degree of pain can be expected after the patient leaves hospital. Most pain can be managed in the first instance by nonopioid drugs.

It is clearly in the patient's best interest if their ongoing management can be overseen by a single prescriber, who can assess ongoing analgesic requirements and make referrals when appropriate. Good communication between the hospital and primary care is essential. $<$

Ross MacPherson has received payments for educational sessions from Seqirus. Gavin Pattullo has received payments for presentations made from Seqirus and Astra Zeneca. 
<ustralian Prescriber

\section{REFERENCES}

1. Gan TJ. Poorly controlled postoperative pain: prevalence, consequences, and prevention. J Pain Res 2017:10:2287-98. https://doi.org/10.2147/JPR.S144066

2. Richebé $P$, Capdevila $X$, Rivat $C$. Persistent postsurgical pain: pathophysiology and preventative pharmacologic considerations. Anesthesiology 2018;129:590-607. https://doi.org/10.1097/ALN.0000000000002238

3. Yang MM, Hartley RL, Leung AA, Ronksley PE, Jetté $N$, Casha S, et al. Preoperative predictors of poor acute postoperative pain control: a systematic review and metaanalysis. BMJ Open 2019;9:e025091. https://doi.org/10.1136/ bmjopen-2018-025091

4. Zeng AM, Nami NF, Wu CL, Murphy JD. The analgesic efficacy of nonsteroidal anti-inflammatory agents (NSAIDs) in patients undergoing cesarean deliveries: a meta-analysis. Reg Anesth Pain Med 2016;41:763-72. https://doi.org/10.1097/ AAP.0000000000000460

5. Australian and New Zealand College of Anaesthetists Position statement on the use of slow-release opioid preparations in the treatment of acute pain. 2018 Mar. www.anzca.edu.au/resources/professional-documents/ statements/sr-opioid-statement-with-disclaimer-20200825 [cited 2020 Nov 1]

6. Australian Bureau of Statistics. 3303.0 Causes of death, Australia, 2018. 2019 Sep 25. www.abs.gov.au/statistics/ health/causes-death/causes-death-australia/latest-release [cited 2020 Nov 1]
7. Lalic S, Ilomäki J, Bell JS, Korhonen MJ, Gisev N. Prevalence and incidence of prescription opioid analgesic use in Australia. Br J Clin Pharmacol 2019;85:202-15. https://doi.org/ 10.1111/bcp.13792

8. Miotto K, Cho AK, Khalil MA, Blanco K, Sasaki JD, Rawson R. Trends in tramadol: pharmacology, metabolism, and misuse. Anesth Analg 2017;124:44-51. https://doi.org/10.1213/ ANE.0000000000001683

9. Fabritius ML, Strøm C, Koyuncu S, Jæger P, Petersen PL, Geisler A, et al. Benefit and harm of pregabalin in acute pain treatment: a systematic review with meta-analyses and trial sequential analyses. Br J Anaesth 2017;119:775-91. https://doi.org/10.1093/bja/aex227

10. Goodman CW, Brett AS. A clinical overview of off-label use of gabapentinoid drugs. JAMA Intern Med 2019;179:695-701. https://doi.org/10.1001/jamainternmed.2019.0086

11. Evoy KE, Morrison MD, Saklad SR. Abuse and misuse of pregabalin and gabapentin. Drugs 2017;77:403-26. https://doi.org/10.1007/s40265-017-0700-x

12. Lavand'homme P, Pogatzki-Zahn E. Chronic postsurgical pain: definition, impact and prevention. Fact Sheet No. 4 on Pain after Surgery. Washington DC: International Association for the Study of Pain; 2017. https://www.iasp-pain.org/ GlobalYear/AfterSurgery 